\title{
Rechtsgeschichte
}

\section{Sven Korzilius}

\section{Besseres Strafrecht?}


wiedergegeben, sind seine Ausführungen in der Kohlrausch-Festschrift von I944: »Ich frage: Wozu haben wir eigentlich Rechtsgeschichte? Müssen wir ganz von vorne anfangen, bloß weil es als >liberalistisch gelten könnte, wenn man auch nur die Diskussion über Aussageerzwingungsmittel, Lügenstrafen und dergleichen für unmöglich hält? «4

Die Autorin begegnet der Weite ihres Themas mit einer staunenswerten Fleißanstrengung. Ihre akribische Spurensuche führte sie in rund zwei Dutzend Bundes-, Landes-, Universitätsund Privatarchive. Sie erhielt Zugang zum Familienarchiv und damit zu Schmidts zahlreichen unveröffentlichten Rede- und Vortragsmanuskripten sowie zu seinem Tagebuch. Als weitere Quelle dienten ihr Interviews mit Zeitzeugen. Für die Universitäts- und Wissenschaftsgeschichte erweist sich die quellengesättigte Abhandlung als aufschlussreich und wichtig, auch kann die Verfasserin mit einigen Trouvaillen aufwarten. Freilich scheint ihr keine Einzelheit zu entlegen, um nicht mitgeteilt zu werden. So werden etwa Namen von Lehrern und Mitschülern ebenso präsentiert wie Schmidts Urteil über die Verpflegung in der amerikanischen Kriegsgefangenschaft (335: "Suppe, die schmeckte, aber nicht satt machte «). Der Umfang der Abhandlung mit ihren nahezu 3000 Fußnoten ist neben der angedeuteten Detailverliebtheit vor allem dem Be-

\section{Besseres Strafrecht?**}

Die ostdeutsche Strafrechtsgeschichte des über achtzigjährigen DDR-Strafrechtslehrers verdient Respekt und Neugier. Bedauerlich ist, dass Buchholz der alten Abwehrattitüde des streben der Verfasserin geschuldet, die Inhalte sämtlicher Publikationen und Vorträge Schmidts in der Reihenfolge ihres Erscheinens vorstellen und bewerten zu wollen. Das intensive Studium älterer Texte mit ihren heute antiquierten Wendungen scheint auf den Duktus der Verfasserin abgefärbt zu haben (»sittliche Persönlichkeitsentfaltung «, »freie Geistigkeit «, »die Aufklärungszeit mit ihrer reichen Geistigkeit und Sittlichkeit «). Wiederholt wird der Leser zudem mit unreflektierten Bewertungen konfrontiert. So habe Schmidt - und dies ausgerechnet mit Blick auf Eduard Dreher - »die Realitätsnähe von Wissenschaftlern (geschätzt), die um das Recht wirklich ringen " (5 I9), bezüglich seiner Tätigkeit als Kriegsgerichtsrat zieht die Verfasserin das Resümee, dass von ihm »kein junger Soldat wegen Fahnenflucht zum Tode verurteilt (wurde), der nicht schon zuvor unangenehm aufgefallen war " (264). Dem Universitätsrektor Schmidt schließlich attestiert sie, dass ihm »das Ansehen der Universität Hamburg in der Öffentlichkeit ein wichtiges Anliegen (war) «(I66), sein späteres Rektorat in Heidelberg verwaltete er »insgesamt pflichtbewusst, fleißig und bescheiden « (407). Leider durchziehen derlei Plattitüden das gesamte Werk und beeinträchtigen die Lesefreude nicht unerheblich.

\section{Arnd Koch}

SED-Staats verhaftet bleibt und durch Treue zum alten Jargon ostalgische Gefühle bedient. Das ostdeutsche wird als das bessere Strafrechtssystem gefeiert. Buchholz erleichtert sich dies,
4 Eberhard Schmidt, Staatsanwalt und Gericht, in: Probleme der Rechtserneuerung. Festschrift für Eduard Kohlrausch zum 70. Geburtstag, Berlin I944, 292 Fn. 98.

\footnotetext{
* Erich Buchrolz, Strafrecht im Osten. Ein Abriss über die Geschichte des Strafrechts in der DDR (Edition Zeitgeschichte 37), Berlin: Homilius 2008, 66I S., ISBN 978-3-89706-857-5
} 
indem er das politische Strafrecht ausklammert ( Io) oder durch die »Verteidigungssituation « des sozialistischen Staats rechtfertigt, so dass er von "zwei Strafrechtssystemen in der DDR « spricht (2 I4) - dem »repressiven politischen « und dem "progressiven normalen «. So gerät eines der Charakteristika des SED-Staats aus dem Blick die Auffassung von der »Justiz als Waffe«, also das instrumentelle Rechtsverständnis, welches insbesondere in der Zeit massiven gesellschaftlichen Umbaus Teile des Strafrechts (»Verbrechen und Klassenkampf «) beherrschte.

Die Darstellung ist zu stark an der normativen Seite orientiert. Politisch-ideologische Texte wie Gesetzespräambeln, kriminalstatistisches Material und Literatur aus der DDR übernimmt Buchholz vorbehaltlos. Großen Wert legt er darauf, dass eine neue, "von alten Traditionen freie Justiz « in der DDR entstand (58), dass man »ein völlig neues Herangehen « im Strafrecht gefunden habe (367). Diese Beschwörung des Neuen entbehrt oft einer realen Grundlage. Innovationsdefizite werden nicht beleuchtet. Ein Beispiel hierfür ist das »Täterstrafrecht «, dessen Ablehnung durch die Wissenschaft Buchholz betont, während er versäumt aufzuzeigen, dass die Rechtswirklichkeit durch Tätertypen geprägt war; und zwar nicht nur im engeren politischen Strafrecht, wo der »Klassenfeind « oder »Republikflüchtling « als Täter und nicht ein einmaliger krimineller Akt im Fokus der Strafverfolgung stand, sondern auch darüber hinaus. Sind »Asoziale«, »Rowdys «, »Rückfalltäter« keine Tätertypen?

Unhaltbar ist die Behauptung, die DDR hätte sich gegenüber Einflüssen des Strafrechts der Sowjetunion weitgehend resistent gezeigt. Entwicklungen des Sowjetstrafrechts wurden wenn auch abgewandelt - zeitnah rezipiert. In den Bereich der Legende gehört ferner, dass
»Einwirkungen des Stalinismus « oft »nicht erkannt (durchschaut) « worden seien (3 I 2).

In den ersten Kapiteln werden die gründlichere Entnazifizierung im Osten, die Volksrichterlehrgänge und die allmähliche Veränderung der sozialen Herkunft der Richter und Staatsanwälte in der DDR sowie die doppelte Staatsgründung behandelt. Der Westen wird für die deutsche Spaltung allein verantwortlich gemacht. Die Ausführungen zu den aus der getrennten Währungsreform entstandenen rechtlichen Problemen (I 37 ff.) und die Darstellung der allmählichen Trennung der Justiz am Beispiel Berlins ( $57 \mathrm{ff}$.) sind nicht uninteressant, aber teilweise zu speziell im Hinblick auf das Hauptthema.

Bezüglich des Jugendgerichtsgesetzes von I952 kritisiert Buchholz zu Recht, dass es die Anwendung von Erwachsenenstrafrecht auf Jugendliche ermöglichte. Im Übrigen fehlt jeglicher Hinweis auf die Schattenseiten des Jugendstrafrechts in der DDR, etwa auf Missstände in Jugendwerkhöfen. Die Strafrechtswissenschaft der DDR habe sich in den fünfziger Jahren kritisch mit Aspekten wie der Äquivalenztheorie, dem Verbrechensaufbau in Tatbestand, Rechtswidrigkeit und Schuld, der normativen Schule und der finalen Handlungslehre sowie der subjektiven Teilnahmelehre auseinandergesetzt. Hier gelingt es dem Autor nicht, überzeugend darzustellen, welche Alternativkonzepte die DDR dem entgegensetzte und warum diese besser gewesen wären. Wie innovativ war es etwa, Franz von Liszts Leitidee des Gesellschaftsschutzes auf die DDR übertragen zu wollen?

Die Gesellschaftlichen Gerichte (Konfliktkommissionen und Schiedskommissionen) waren für Buchholz »ein geeigneter und humaner Weg zur Vorbeugung und Bekämpfung der Kriminalität « (347). Sie müssen aber als Teilelement 
der Erziehungsdiktatur verstanden werden. In ihrer praktischen Konsequenz bildeten sie die erste Instanz gesellschaftlicher Etikettierung und Ausgrenzung und den Einstieg in eine härtere, formalere Verfolgung.

Ein gewisses Verständnis für das Problem der Erziehungsdiktatur zeigt Buchholz im Bereich »asozialen Verhaltens". Die Regelung im Wege der Verordnung vom 24. August I96I (Arbeitserziehung bei »Arbeitsscheu «) sei verfassungswidrig gewesen, die Organisation der Arbeitserziehungslager mangelhaft (347). Paragraph 249 des StGB von 1968 wird als » massive und weit gefasste Strafbestimmung " kritisiert; dabei wird anerkannt, dass die DDR hier der Strafrechtstradition verhaftet blieb (388ff.) eine fast schon überraschende Annäherung an Positionen des (von Buchholz auf eine Liste mit »der DDR fremd oder gar feindlich gegenüberstehender Personen « gesetzten) Rezensenten.

Die Teilnahme der Bevölkerung an der Strafrechtsreformdiskussion, welche in das neue StGB von 1968 mündete, bewertet Buchholz über (4I I) und betont die »eigenständigen Wurzeln « der DDR zu stark. Bahnbrechende Innovationen waren nicht zu verzeichnen. Nur einzelne Regelungen waren fortschrittlich, etwa die Abschaffung des Eides (409) oder der Wegfall der Pflicht des Verurteilten, die Verfahrenskosten zu tragen (434).

Buchholz sieht zutreffend die Verschärfung der Strafpolitik unter Honecker, die Zunahme gerichtlicher Verfahren zu Lasten der Verfahren vor Gesellschaftlichen Gerichten sowie die $\mathrm{Zu}$ nahme von Freiheitsstrafen zu Lasten weicherer Sanktionen. Mit seiner Kritik an den Strafrechtsänderungsgesetzen von 1972, I974, I977 und I979 (die Verschärfungen enthalten) ist er zu zurückhaltend (460). Bezüglich der Strafgefangenenquote wird anerkannt, dass sie (anders als die Kriminalitätsquote) schlechter war als die der Bundesrepublik. Auch eine Überbelegung der Haftanstalten gesteht Buchholz ein, ebenso die Tatsache, dass in den siebziger Jahren die »Kontrollelemente noch weiter ausgebaut « wurden (476 f.) und dass Probleme »der sozialen Integration von Menschen zu lange nicht als solche behandelt « wurden, "sondern vorrangig unter sicherheitspolitischen Aspekten " (477) so dass Straffälligkeit »konserviert und reproduziert « wurde.

Es hätte darauf hingewiesen werden müssen, dass damit das DDR-Strafrecht immer täterbezogener geworden war, ebenso hätte die Strafrechtsproblematik der DDR stärker mit der Sozialgeschichte der DDR in Verbindung gebracht werden sollen. Innere strukturelle Probleme der DDR behandelt Buchholz nicht, interpretiert vielmehr Strafrechtsverschärfungen allein mit »Kaltem Krieg «, »ideologischer Diversion « und "psychologischer Kriegsführung « seitens des Westens. Auch für die Republikfluchtproblematik macht er allein den Westen verantwortlich, seine Haltung gegenüber Ausreisewilligen (626f.) bleibt der DDR-offiziellen Sichtweise von vor 1989 treu. Während er für die DDR das politische Strafrecht ausklammert oder rechtfertigt, konzentriert sich Buchholz bei seinem vergleichenden Blick auf die Bundesrepublik fast ausschließlich auf die politisch motivierte Strafverfolgung und Gesetzesverschärfung.

Kritisches Potential liegt in Buchholz' "Zweifel am Sinn und an einer Wirksamkeit von Strafrecht und Strafe« (52I). Hätte er seine Geschichte des DDR-Strafrechts konsequent aus dieser strafrechtskritischen Perspektive geschrieben, wäre sie über weite Passagen anders ausgefallen. Statt ihrer vermeintlichen Einmaligkeit (645) hätte der Autor dann betont, wie wenig die DDR aus dem Rahmen konservativer, zum 
Teil über viele Jahrhunderte gewachsener Strafrechtssysteme ausscherte. Eine grundsätzliche Infragestellung des herkömmlichen Strafsystems ist in der DDR nicht gewagt worden. Gegen- wärtig scheinen solche Ideen indes weniger diskutierbar zu sein denn je.

Sven Korzilius

\section{Anwaltsgeschichte aus Anwaltssicht*}

Ein fast 700 Seiten starkes und roo Euro teures Werk, das laut Einleitung die »Entwicklung der deutschen Anwaltschaft und ihres politischen, wirtschaftlichen und gesellschaftlichen Umfeldes vom 8.5.I945 an näher betrachten « (33) möchte, verfasst von einem früheren Präsidenten des Deutschen Anwaltvereins (DAV): Verdienen Buch und Autor nicht mehr als das obligatorische Lob der (früheren) Funktionärskollegen in den einschlägigen Gazetten ${ }^{\text {I }}$ Vermutlich ist die Skepsis der historischen Zunft groß, wenn von einem Rechtsanwalt, dazu noch einem ehemaligen Verbandsfunktionär, eine Nachkriegsgeschichte des eigenen Berufsstandes präsentiert wird. Busse knüpft damit explizit an seine Kollegen Fritz Ostler ${ }^{2}$ und Adolf Weißler ${ }^{3}$ an, deren vor fast $30 \mathrm{bzw}$. mehr als Ioo Jahren erschienene Monographien - unbeschadet ihrer nach wie vor überragenden Bedeutung als Nachschlagewerke - aus heutiger Sicht unbestreitbare wissenschaftliche Defizite aufweisen. Ist die Skepsis also begründet?

"Ja und nein « lautet die Antwort des Rezensenten, der allerdings, was nicht verschwiegen werden soll, auch zum anwaltlichen Kollegenkreis gehört: Busses Buch ist ein sorgfältig recherchiertes, insgesamt äußert informatives und - bis auf einige sprachliche Mängel - ansprechend geschriebenes Werk, das seinen Lesern einen guten Überblick verschafft und alle wich- tigen Entwicklungen im Bereich der Berufsausübung und -politik vor und nach I990 ausführlich nachzeichnet. Die einschlägige Literatur ist weitestgehend berücksichtigt, Archivmaterial wurde ebenfalls ausgewertet, ${ }^{4}$ zum besseren Verständnis der Entwicklungen im Ostteil Deutschlands hat Busse Zeitzeugeninterviews geführt. Dennoch wird es dem oben zitierten selbst gesetzten Anspruch nicht ganz gerecht. Dazu später mehr.

Zunächst ein Blick auf den Inhalt: Zeitlichchronologisch beschreibt Busse die »Entwicklungen in Ost und West «, wie es im Untertitel seines Werkes heißt; erst in den Westzonen I945-I949 und der Bundesrepublik bis I990, dann in der sowjetischen Besatzungszone und in Berlin bis I953, schließlich in der DDR von I953 (Zeitpunkt der Kollektivierung) bis I990 und zu guter Letzt seit der deutschen Einheit bis in die Gegenwart. Dabei fällt auf, dass das Kapitel über die DDR am umfangreichsten ist. Zwar ist Busse ohne weiteres zuzugeben, dass diese historische Phase zentraler Bestandteil der (deutsch-) deutschen Anwaltsgeschichte ist. Sein Bemühen, durch die Befragung von Zeitzeugen die Authentizität der Darstellung zu vergrößern, verdient durchaus Anerkennung (auch wenn Busse damit Kritik an deren Auswahl geradezu herausfordert ${ }^{5}$ ). Jedoch verschiebt diese Ausführlichkeit die Proportionen in einer Weise
* Felix Busse, Deutsche Anwälte. Geschichte der deutschen Anwaltschaft I945-2009, Berlin: Duncker \& Humblot 2010, 677 S., ISBN 978-3-428-I3282-9

I Hartmut Kilger, Keine Nostalgie - sondern Erkenntnisgewinn, in: Anwaltsblatt 2010, I 24 (Kilger wurde fünf Jahre nach Busse DAVPräsident) bzw. Eberhard HaAs, Für Recht und Freiheit, in: Mitteilungen der Bundesrechtsan- waltskammer 20I0, I9 (Haas war von I99I bis I999 Präsident der BRAK).

2 Fritz Ostler, Die deutschen Rechtsanwälte I87I-I97I, 2. Aufl. Essen I982.

3 Adolf Weissler, Geschichte der Rechtsanwaltschaft, Leipzig 1905 , Nachdruck Frankfurt I967.

4 Dies erschließt sich aus den Fußnoten. Leider enthält das Literaturverzeichnis keine Auflistung der Archive mit den jeweils genutzten Beständen.

5 Vgl. 65 I ff: Fast alle waren, viele davon an exponierter Stelle, als Anwälte in der SBZ/DDR tätig; erstaunlicherweise fehlt Günter Kröber, I96I aus der Anwaltschaft wegen staatsfeindlichen Verhaltens ausgeschlossen, I990 rehabilitiert und 200I zum Präsidenten der Rechtsanwaltskammer Sachsen gewählt. 